\title{
Allopatric integrations selectively change host transcriptomes, leading to varied expression efficiencies of exotic genes in Myxococcus xanthus
}

Li-Ping Zhu, Xin-Jing Yue, Kui Han, Zhi-Feng Li, Lian-Shuai Zheng, Xiu-Nan Yi, Hai-Long Wang, You-Ming Zhang and Yue-Zhong Liं

\begin{abstract}
Background: Exotic genes, especially clustered multiple-genes for a complex pathway, are normally integrated into chromosome for heterologous expression. The influences of insertion sites on heterologous expression and allotropic expressions of exotic genes on host remain mostly unclear.

Results: We compared the integration and expression efficiencies of single and multiple exotic genes that were inserted into Myxococcus xanthus genome by transposition and attB-site-directed recombination. While the sitedirected integration had a rather stable chloramphenicol acetyl transferase (CAT) activity, the transposition produced varied CAT enzyme activities. We attempted to integrate the 56-kb gene cluster for the biosynthesis of antitumor polyketides epothilones into M. xanthus genome by site-direction but failed, which was determined to be due to the insertion size limitation at the attB site. The transposition technique produced many recombinants with varied production capabilities of epothilones, which, however, were not paralleled to the transcriptional characteristics of the local sites where the genes were integrated. Comparative transcriptomics analysis demonstrated that the allopatric integrations caused selective changes of host transcriptomes, leading to varied expressions of epothilone genes in different mutants.
\end{abstract}

Conclusions: With the increase of insertion fragment size, transposition is a more practicable integration method for the expression of exotic genes. Allopatric integrations selectively change host transcriptomes, which lead to varied expression efficiencies of exotic genes.

Keywords: Transposition, Site-directed integration, Chloramphenicol acetyl transferase, Epothilone biosynthetic gene cluster, Expression efficiency, Transcriptome, Myxococcus xanthus

\section{Background}

Heterologous expression is a routine laboratory technique to obtain massive quantities of interesting products, not only single recombinant proteins but also metabolites from complex biosynthetic pathways, such as microbial secondary metabolites. Efficient expression and high yields of exotic genes in acclimatized

\footnotetext{
*Correspondence: lilab@sdu.edu.cn

State Key Laboratory of Microbial Technology, School of Life Science, Shandong University, Jinan 250100, China
}

hosts are not only important for increasing the yields of desired products, but also useful in the discovery of novel compounds, for example, the products of those cryptic secondary metabolic pathways $[1,2]$. Heterologous expressions of single gene products can normally be achieved in high efficiency and yields in Escherichia coli after codon optimization, promoter selection, co-expression with chaperon protein genes, and/or cultivation improvement [1-3]. However, heterologous expressions of metabolites from pathways containing cluster-organized multiple-genes confront much more difficulties and 
limits [4]. Researchers have to establish suitable transfer systems for large-sized gene clusters, maintain integrity of well-organized genes during transfer, trigger efficient expressions of multiple genes, provide sufficient supplies of substrates for the biosynthesis, facilitate secretion to eliminate products-feedback inhibition on biosynthesis or toxic effects on host, and reduce negative effects of local metabolisms on the desired expressions. Up to now, efficient expression of a complicated biosynthetic pathway in heterologous host is still a challenge.

Because of large size, multiple genes of complicated biosynthetic pathways are often integrated into chromosome for heterologous expression. For example, antitumor compounds epothilones were originally isolated from myxobacterial Sorangium cellulosum cultures [5]. The 56-kb biosynthetic gene cluster has been successfully introduced into different hosts, including Streptomyces coelicolor [6, 7], S. venezuelae [8], E. coli [9], Pseudomonas putida [10] and other myxobacterium Myxococcus xanthus [10-12]. While the yields of epothilones in M. xanthus might be up to $160 \mu \mathrm{g} / \mathrm{L}-$ titer [11], the yields in those distantly related hosts were lower than $1 \mu \mathrm{g} / \mathrm{L}$, or undetectable $[9,10]$, probably due to incompatibility of the exotic DNA and/or metabolites. Thus, although distantly related hosts may have merits in genetic performances, growth and fermentation, closely related species turn to be preferable for heterologous expression of the products from large gene clusters. However, the productions of epothilones are also greatly varied in those engineered Myxococcus strains, even with almost identical genetic backgrounds [10, 11], suggesting internal uncertainty for the expression of multiple exotic genes. For example, it is yet unclear whether and how insertion sites influence the expression efficiency of exotic genes and whether and how allotropic expressions influence on host cells.

Transposition is able to bring exotic genes into host chromosome randomly. Recently, transposon technique has been developed for heterologous expression of largesized gene clusters [10], which provides an approach for analysis of the influences of integration patterns. In this study, we compared expression efficiencies of the chloramphenicol acetyl transferase gene that was introduced into chromosome via transposition insertion or site-specific insertion. We then constructed vectors containing the entire 56-kb epothilone gene cluster, stitched using a modified recombination strategy, for one-step introduction into $M$. xanthus cells. We assayed effects of integration sites on the yields of epothilones and transcriptome changes in different mutants. Our results indicated that allopatric integrations selectively change host transcriptomes, leading to varied expression efficiencies of exotic genes in M. xanthus.

\section{Results and discussion}

Site-specific and transposition insertions of cat gene in $M$. xanthus

There are usually two ways to introduce exotic genes into $M$. xanthus genome. One is arbitrary insertion via transposon such as miniHimar1, a plasmid derived from the mariner element Himar1 [13, 14], while the other is site-specific recombination, normally at the chromosomal attB site via $M x 8$ att, developed from myxophage $M x 8$ DNA $[15,16]$. To test whether insertion pattern influences heterologous expression, we introduced the chloramphenicol acetyl transferase (cat) gene into the genome of $M$. xanthus DZ2 via either transposition (Tp) or site-directed insertion (Mx8). Previous studies related to heterologous expressions of the epothilone biosynthetic gene cluster normally employed an exotic promoter like aphII, the kanamycin promoter in pKK-aphII [17], which is frequently used in M. xanthus $[18,19]$. We once reported that the 843-bp epoP promoter for the biosynthetic gene cluster of epothilones from $S$. cellulosum So0157-2 exhibited much higher activities than aphII in E. coli [17]. Thus, four plasmids containing the respective promoter and integration elements, i.e. pTp-epoP, pMx8-epoP, pTp-aph and pMx8-aph, were constructed (Additional file 1: Figure S1), which were further electroporated into the $M$. xanthus DZ2 strain, respectively.

Compared with the wild type strain, the cat expression had small effects on the growth of recombinant cells (Figure 1a). We picked-up 20 colonies randomly from each of the four recombinant categories to assay their chloramphenicol acetylation activities. The site-directed recombination produced rather stable CAT activities, i.e. approximately 450 and $100 \mathrm{pg} / \mu \mathrm{g}$ in the pMx8-epoP and pMx8-aph transformants, respectively. However, the pTp-epoP and pTp-aph strains showed highly varied CAT activities, ranging from 209.31 to $638.49 \mathrm{pg} / \mu \mathrm{g}$ in the pTp-epoP transformants or from 55.59 to $168.24 \mathrm{pg} /$ $\mu \mathrm{g}$ in the pTp-aph transformants (Figure 1b). Furthermore, it is clear to see that the $M$. xanthus transformants from the pTp-epoP or pMx8-epoP plasmids exhibited several times higher CAT activities than those from the corresponding $\mathrm{pTp}$-aph or $\mathrm{pMx} 8$-aph plasmids. The epoP promoter is also more efficient than the aphII promoter in $M$. xanthus. Accordingly, the original epoP promoter was used for further heterologous expressions of the epothilone biosynthetic genes.

\section{Construction of plasmids containing the entire epothilone biosynthetic gene cluster}

Epothilones are biosynthesized by a gene cluster of seven multifunctional modules, spanning approximately $56 \mathrm{~kb}$ in length $[20,21]$. In this study, we constructed the whole epothilone biosynthetic genes into single plasmids for the 

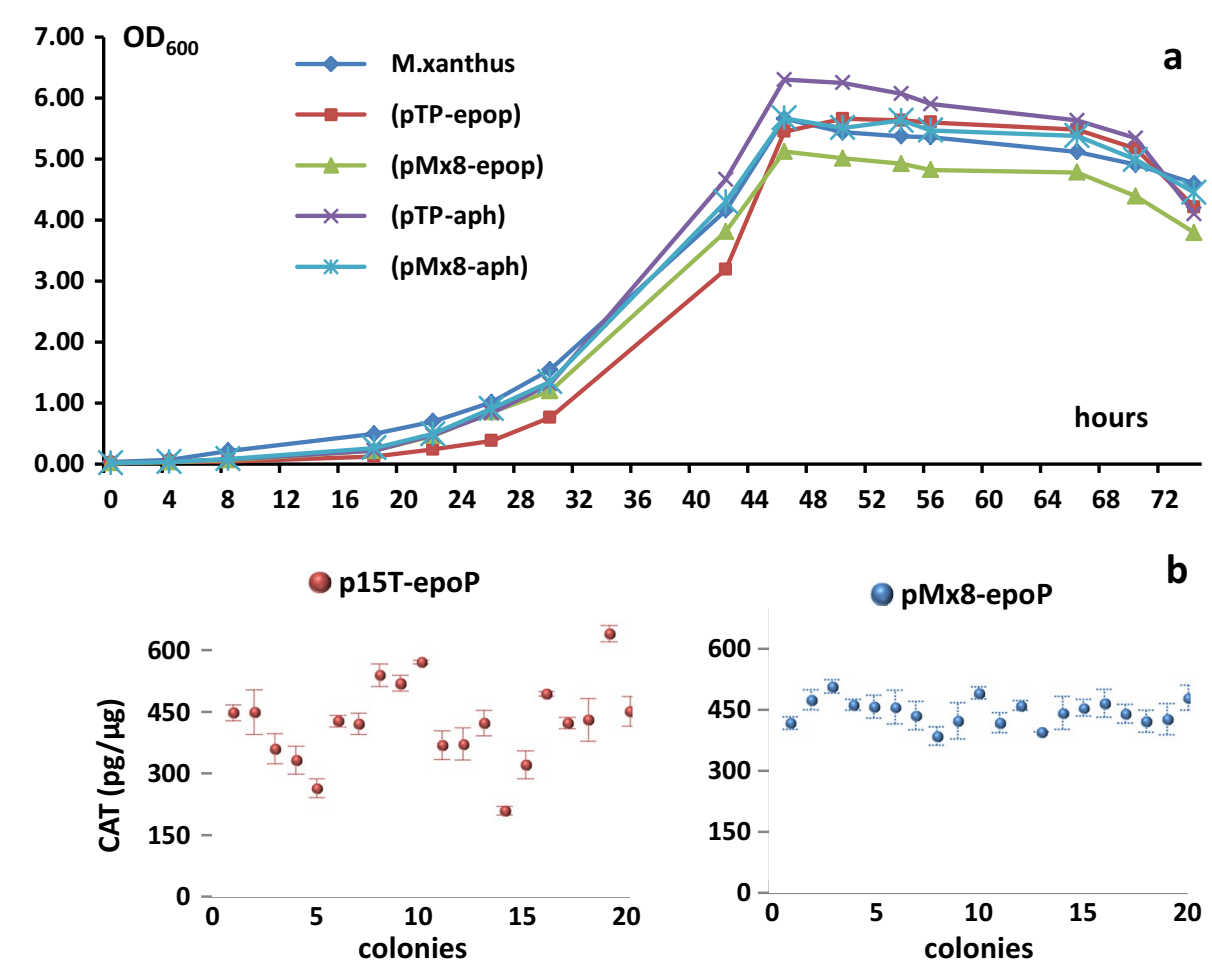

150 -
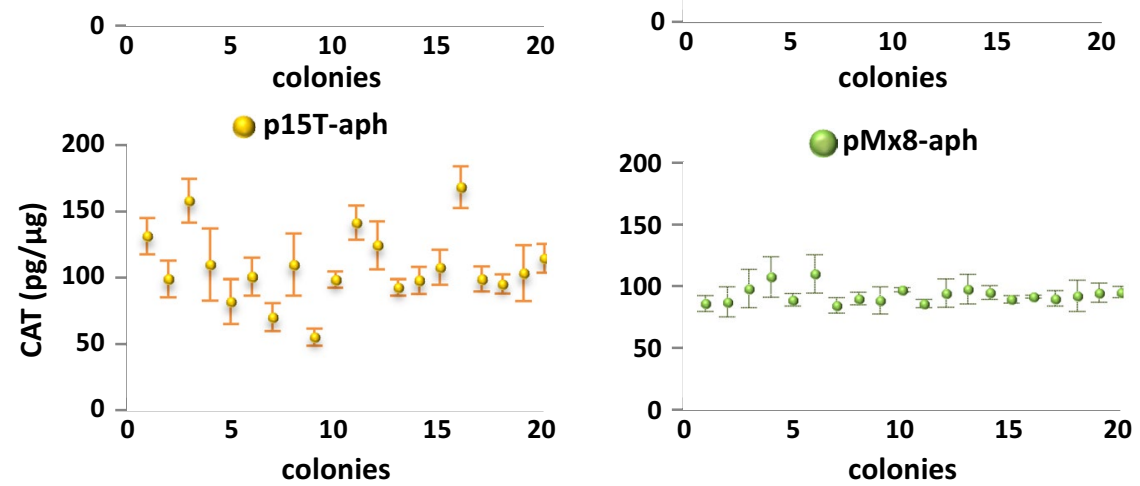

Figure 1 Growth and CAT activities of M. xanthus transformants integrated from the plasmids pTP-epoP, pMx8-epop, pTP-aph or pMx8-aph. a The growth curves of M. xanthus transformants, compared with M. xanthus DZ2. b The CAT expression activities in M. xanthus transformants. Twenty clones were selected randomly from each of the four recombinant categories.

integration in M. xanthus chromosome. The epothilone genes were from the Cosmid10 and Fosmid3B11 plasmids, which separately contained a 38.5-kb fragment from epoA to the front part of epoD and a 34.4-kb fragment from the rear part of $e p o C$ to the downstream fragment of epoF. The two fragments overlapped a $6.5-\mathrm{kb}$ region.

To simplify recombination and to avoid false-junction, we stitched the two fragments into a complete epothilone gene cluster using a one-stop-way strategy. The homologous arms included an upstream arm, a downstream arm and a shared middle arm. The shared middle arm was designed to stitch the two large fragments, permitting the two rounds of recombination happening in no particular order for integration of the epothilone gene cluster fragments into plasmid. The three homologous arms were constructed into a single vector, and the stitching was carried out in E. coli GB05-red by means of the red-ET recombination system (diagrammed in Figure 2a). The correct colonies were screened by the negative marker galk and $s a c B$, and the junction regions were verified by restriction digestion (Figure $2 \mathrm{~b}$ ). After sequencing verification, we got the correct recombinant plasmid p15A-Tp-CF (68.8 kb).

We also constructed a recombinant plasmid containing the $M x 8$ att sequence (Figure 2a, b) for site-specific recombination.

\section{Integration of the epothilone biosynthetic genes into $M$. xanthus chromosome}

The plasmids were subject to one-step integration into $M$. xanthus DZ2 chromosome, and the recombinant 


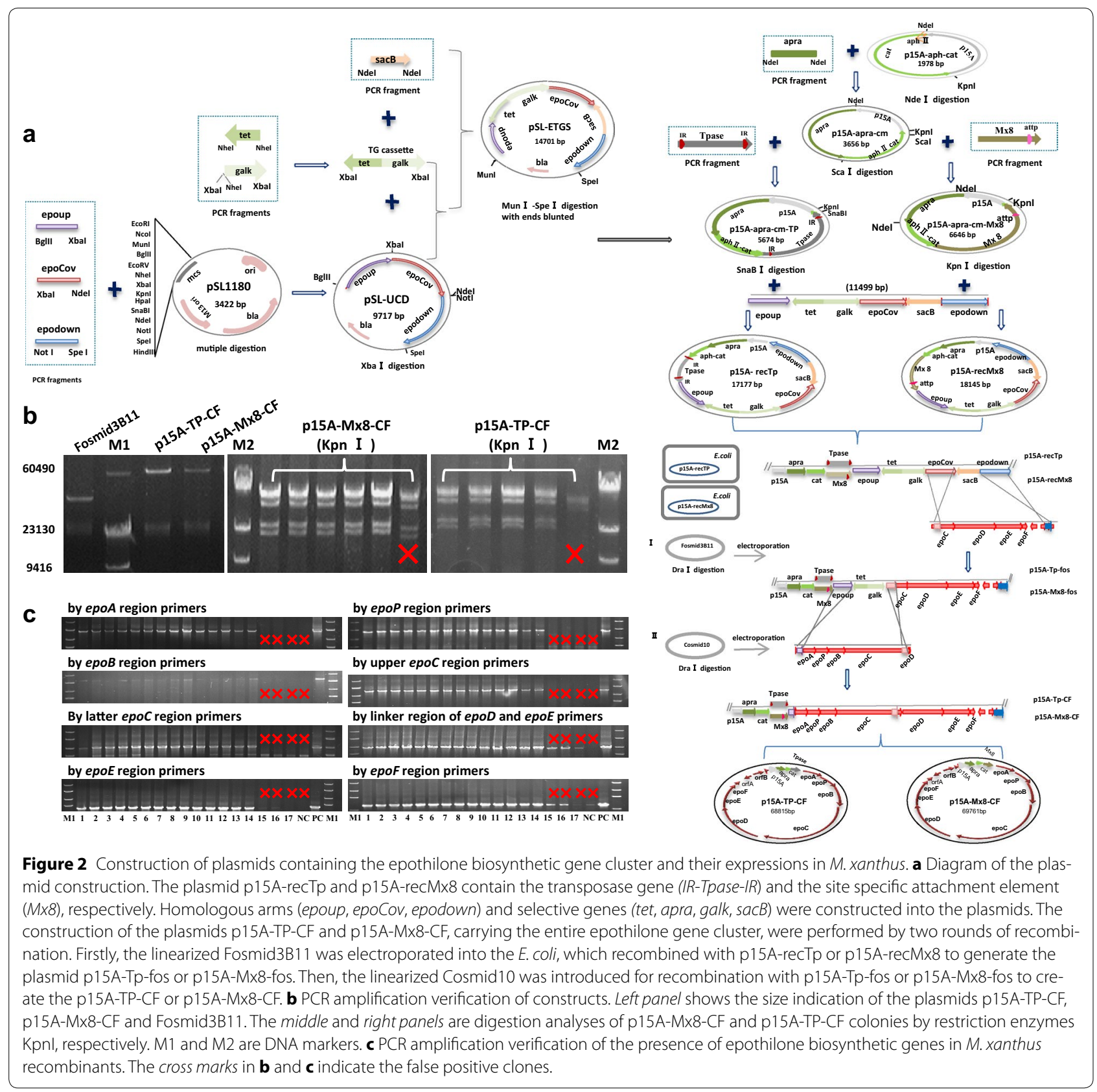

colonies were screened by selection markers Apra and $\mathrm{Cm}$. After 6-8 days of incubation at $30^{\circ} \mathrm{C}$, we obtained 21 colonies from the CYE plate plus the Apra and $\mathrm{Cm}$ antibiotics in a single transformation performance. PCR amplification determined that 14 of the 21 antibioticsresistant colonies were correct transformants, while the other seven contained partial or none of epothilone genes. A further transformation performance of p15ATp-CF in M. xanthus DK1622 strain yielded 30 correct transformants from 59 antibiotics-resistant colonies. We also performed transformations in SW504, a difA mutant of $M$. xanthus DK1622 [22, 23], from which 4 correct transformants were obtained. Figure 2c demonstrates some PCR amplification results. Our results showed that the transposition technique was highly efficient to nail large exotic genetic fragments into the chromosome of different $M$. xanthus strains.

Electroporation of the p15A-Mx8-CF plasmid with M. xanthus DZ2 strain obtained six tiny colonies on the selection plate, which, however, were turned out to be 
spontaneous mutants without any epothilone genes. We performed transformation of the plasmid in different $M$. xanthus strains, which also failed in obtaining any correct transformant. To testify whether the failure was due to the size limitation for integration at the $M x 8$ att site, we constructed smaller plasmids of $42.0-\mathrm{kb}$ p15A-Mx8-fos, derived from the recombination of the linear fosmid3B11 with $\mathrm{p} 15 \mathrm{~A}-\mathrm{recMx} 8$, and $18.1-\mathrm{kb} \mathrm{p} 15 \mathrm{~A}-\mathrm{recMx} 8$. The transformation performance with these two plasmids under the same conditions produced 4 and 47 correct colonies in M. xanthus DZ2, respectively. We thus concluded that the recombination efficiency of the $M x 8$ att site decreased sharply with the increase of the integration fragment sizes.

\section{Epothilone production abilities and integration sites in the transposition transformants}

We assayed the production abilities of epothilones in 48 correct recombinant transformants derived from different $M$. xanthus strains, i.e. DZ2, DK1622 or SW504. The strains were cultivated in the CYE medium for 5 days of incubation, and the production of epothilones was characterized by high performance liquid chromatographymass spectrometry (HPLC-MS) techniques. The results showed that all these recombinants were able to produce epothilones A and B; whereas no epothilone was detectable in the original M. xanthus strains (Figure 3a; detailed information of the yields in different recombinants is

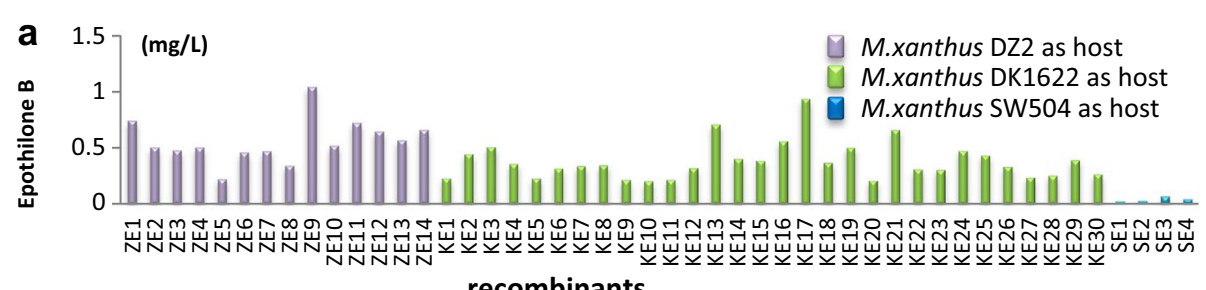

recombinants
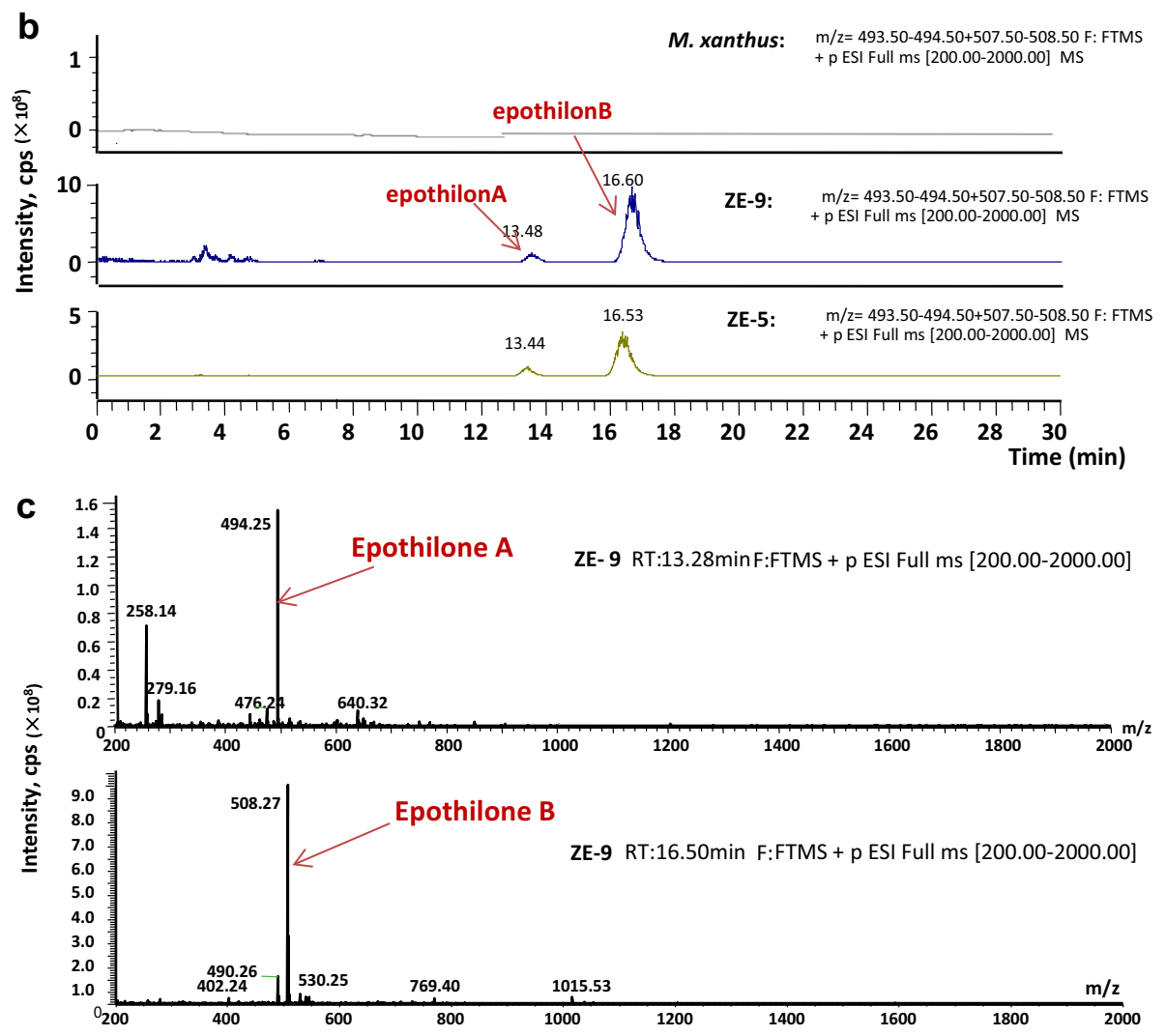

Figure 3 Production of epothilone B in the M. xanthus transformants (a) and HPLC-MS detection (b, c). a The epothilone productions, assayed after 7-days fermentation. ZE-, KE- and SE- series strains are termed for those recombinants from M. xanthus DZ2, DK1622 and SW504 strains, respectively. b HPLC results of the ion chromatograms of HPLC-MS runs. The upper row, a negative control form the wild type M. xanthus DZ2. The middle and the lower rows demonstrate the results from ZE-5 and ZE-9, respectively. c The fragmentation patterns of epothilone A and epothilone B produced in the isolate no. 9 with the correct molecular weight minus the $\mathrm{H}^{+}$(epothilone $\mathrm{A}=493$; epothilone $\mathrm{B}=507$ ). 
provided in Additional file 2: Table S1; Figure 3b, c demonstrate the HPLC-MS detection of epothilones production). Similar to that of the CAT enzyme activities in transposition transformants, the yields of epothilones varied significantly in these recombinants. For example, in the M. xanthus DK1622 host, the yields in CYE medium ranged from 0.045 to $0.17 \mathrm{mg} / \mathrm{L}$ of epothilone A and $0.22-1.05 \mathrm{mg} / \mathrm{L}$ of epothilone B. Among the three employed $M$. xanthus hosts, the yield ranges were similar in the DZ2 and DK1622 mutants, but much low in the SW504 mutants. The production abilities of epothilones in M. xanthus transposition recombinants from DZ2 or DK1622 were rather similar to that in those wild type epothilone-producing S. cellulosum strains [24]. However, in contrast to the 2:1 ratio of epothilones A to B or almost single A without B in S. cellulosum strains [24], the ratios of epothilones A-B produced in these $M$. xanthus producers were all approximately 1:5 (Additional file 2: Table S1). Compared to those previously reported epothilone-producing $M$. xanthus recombinants (approximately $0.1 \mathrm{mg} / \mathrm{L}$ or less) $[10,11]$, some of the transposition transformants gave much higher productions under unimproved fermentation conditions.

We mapped the integration sites of the epothilone genes in genome using the genome walking technique. Sequencing revealed that the epothilone genes were located at 41 different sites in the 48 recombinants, of which six were repeatedly inserted (Figure 4; details are provided in Additional file 2: Table S1). The epothilone genes were all in the same direction, and these integration sites scattered along the Myxococcus chromosome, seemly with no bias. The recombinants with the same integration sites also had similar yields of epothilones, which was consistent with that of the site-directed insertion of CAT gene at the attB site (Figure 1b). However, no clear correlation was observed between the production abilities of epothilones and the insertion sites in genome (Figure 4).

\section{Transcriptomics variation in epothilone-producing transposition transformants}

The above results suggested that the production differences probably resulted from the influences of insertion sites on the expressions of the epothilone genes. In order to investigate how allopatric integrations influenced the yields of epothilones, we assayed the transcriptomes in three recombinants, as well as wild type strain DZ2. The three recombinant strains, i.e. ZE-5 (inserted at MXAN_7320), ZE-9 (inserted at MXAN_5011), and ZE-14 (inserted at MXAN_4403), were varied of their yields of epothilones in the CYE medium $(0.22 \pm 0.08$, $1.05 \pm 0.21$ and $0.66 \pm 0.09 \mathrm{mg} / \mathrm{L}$ epothilone $\mathrm{B}$, respectively; Additional file 2: Table S1). To confirm the total biosynthetic genes were at the same place in each strain, we performed PCR amplification of the junction regions between the seven modules of the gene cluster. The results showed that all the modules were neighbored in each of these three recombinants (Additional file 3: Figure S2). Growth assays indicated that the three strains had similar growth curves as the wild type strain (Figure 5a). The RNA materials were extracted from the cells, which were collected when XAD-16 resin was harvested for the assay of epothilone production. After confirmation of the extracts free of DNA, we sequenced transcriptomes using strand specific RNA-seq technique (Illumina Miseq 2000 technique). The sequencing obtained 18.3, 21.3, 18.5 and 20.8 million reads for DZ2, ZE5, ZE9 and ZE14, respectively. Totally, 97.4-98.2\% of reads were mapped to genome, and the distribution had good correlation with the gene number in each strand. We checked the expression levels of the insertion-mutated genes in the wild type strain DZ2 (Additional file 2: Table S1), which, however, showed no correlations with the production abilities in the transposition recombinants.

Comparative transcriptomes showed similar distributions of expression density of the total genes in the four recombinants (Figure 5b). The expression patterns of the epothilone genes varied significantly in the three recombinants (Figure 5c), but parallel to their production abilities. The expression levels of the epothilone biosynthetic genes in ZE- 5 were low, especially the latter part of this big gene cluster. In contrast, the epothilone genes expressions in ZE-9, which had approximately five times of the epothilone production in ZE-5, were rather high in different regions in the gene cluster. In the ZE-14 strain, although expressions of the front region were much high, the hinder genes had sharply decreased expressions, which probably led to the medium level of the epothilone production.

Compared to the M. xanthus DZ2 strain, many Myxococcus-own genes were up- or down-regulated distinctly in these allopatric insertion mutants (Additional file 4: Table S2). For example, three hypothetical protein genes (MXAN_5126, MXAN_5530 and MXAN_3967) were up regulated, while two genes (MXAN_4480 predicted for a DNA binding protein and MXAN_4324 for a hypothetical protein) were down regulated in the ZE-9 and ZE-14 strains. Six genes were specifically down regulated of their expressions in the ZE-9 strain, but were not significantly changed in ZE- 5 or ZE-14. In all the three tested recombinant strains, three Myxococcus genes, i.e. MXAN_1576 (predicted encoding a major facilitator family transporter), MXAN_1093 (a DNA-binding response regulator) and MXAN_7163 (bis (5'-nucleosyl)tetraphosphatase, symmetrical), were found to be significantly up-regulated, while two genes, i.e. MXAN_7372 


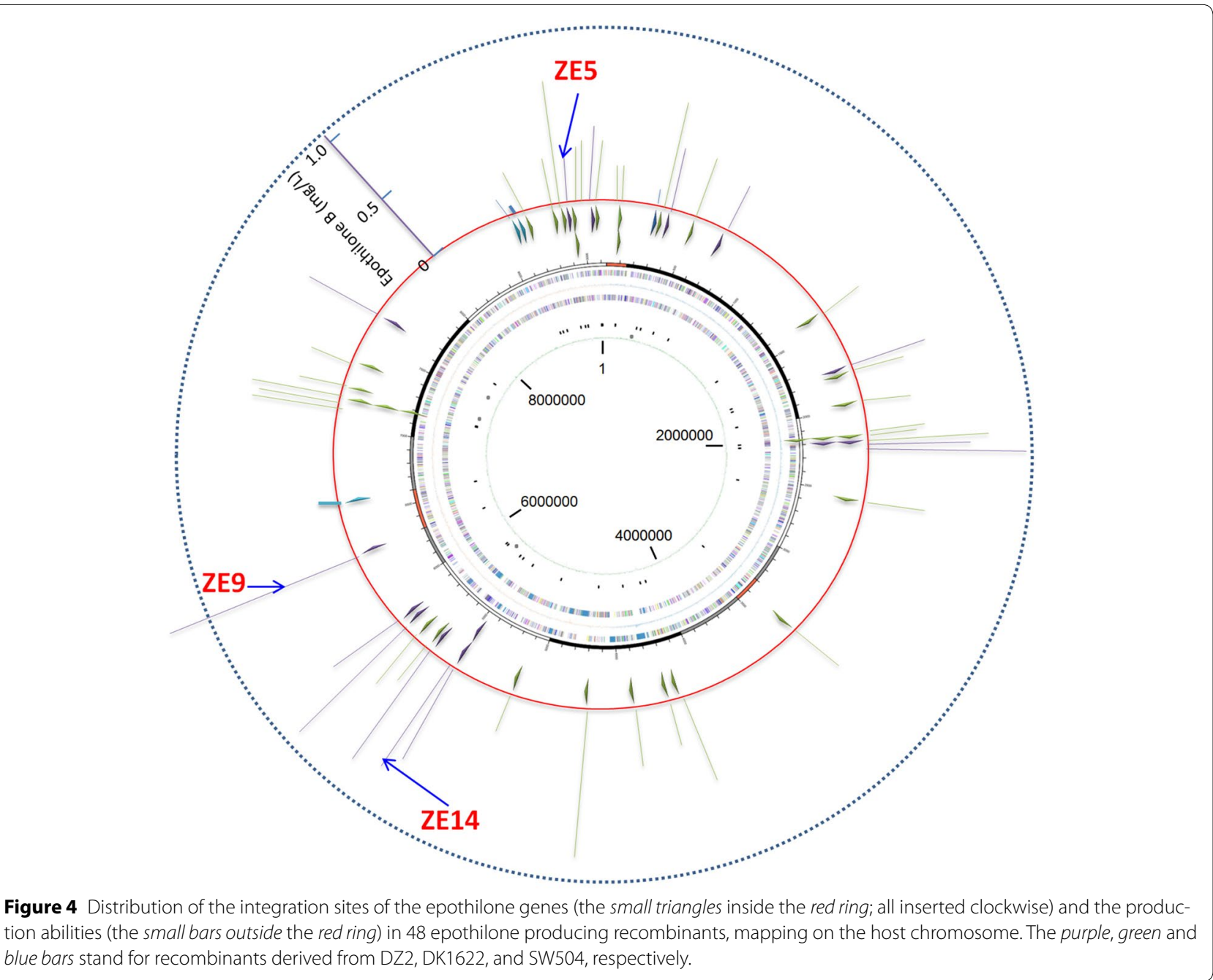

(hypothetical protein) and MXAN_4372 (DNA-binding protein) were significantly down regulated. It is noted that the locations of these up- or down- regulated genes normally had no relationship with the insertion sites in Myxococcus genome. These transcriptome changes indicated that allopatric integration of exotic genes in genome had some specific effects on the transcriptome in host, which probably resulted in distinct expressions of the exotic genes.

\section{Conclusions and outlook}

In contrary to potential size-limitation of the inserted fragments at directed sites, demonstrated using the attB site in $M$. xanthus, transposition attempts to position large-sized genetic materials randomly into genome, thus provides much more opportunities for the integration of exotic genes. Such random transposition integration is blind, and the introduced exotic genes may inactivate or disturb functions of host genes, but also yields varied expression levels of exotic genes. The expression differences of allopatrically integrated genes did not follow the local expression characteristics, but rather the results of some global changes of the host transcriptome. We might be able to improve the expressions of the exotic genes further by genome engineering based on the transcriptome changes. In addition, the expressions of epothilone genes were rather different in different cluster regionsincreasing the expression of low-expressed genes will be also useful for the yield improvement.

\section{Methods}

\section{Bacterial strains, plasmids and culture conditions}

Bacterial strains and plasmids used in this study are listed in Table 1. Escherichia coli DH5 $\alpha$ was used for routine transformations and sub-cloning. E. coli GB05-red, a derivative of DH10B [25], was used in recombination performance. E. coli strains were grown routinely in Luria Broth (LB) medium (10 g/L peptone, $10 \mathrm{~g} / \mathrm{L}$ yeast extract 


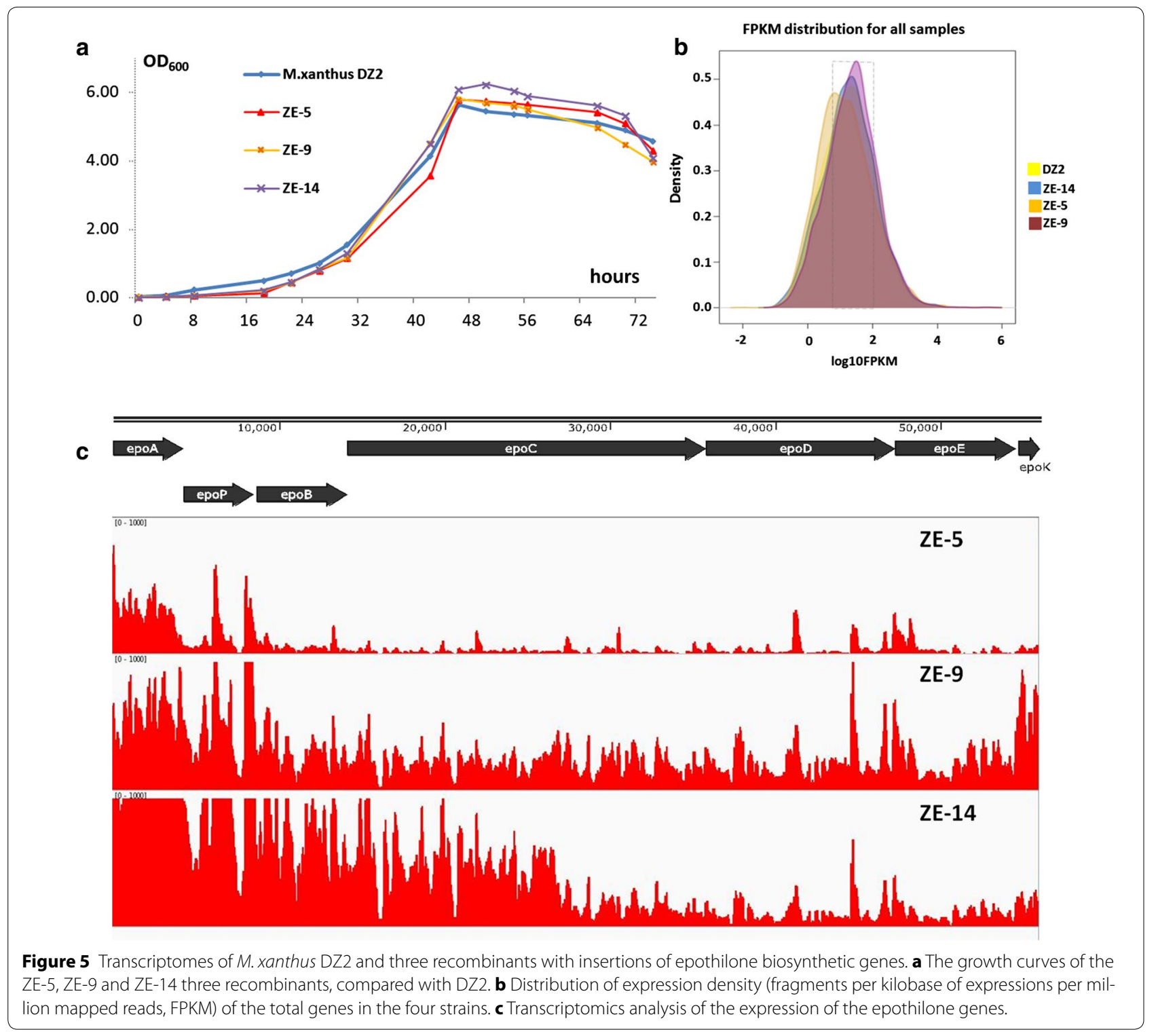

and $5 \mathrm{~g} / \mathrm{L} \mathrm{NaCl}$; pH7.2), and Myxococcus xanthus strains were grown in CYE medium $[10 \mathrm{~g} / \mathrm{L}$ casitone, $5 \mathrm{~g} / \mathrm{L}$ yeast extract, $10 \mathrm{mM}$ 3-(N-morpholino) propanesulfonic acid (MOPS; pH 7.6) and $4 \mathrm{mM} \mathrm{MgSO} 4$ ] [26]. The growth temperatures were $37^{\circ} \mathrm{C}$ for $\mathrm{E}$. coli and $30^{\circ} \mathrm{C}$ for $M$. xanthus, respectively. For selection of constructed plasmids or transformants, different antibiotics, i.e. ampicillin [Amp], $100 \mu \mathrm{g} / \mathrm{mL}$; kanamycin [Km], $40 \mu \mathrm{g} / \mathrm{mL}$; chloramphenicol $[\mathrm{Cm}$ ], $10 \mu \mathrm{g} / \mathrm{mL}$; or apramycin [Apra], $25 \mu \mathrm{g} / \mathrm{mL}$, were added in LB or CYE media.

\section{Construction of CAT reporter vectors}

We amplified the 836-bp replicon $p 15 A$ from pACYC184 using the primer pair of P15A. The chloramphenicol acetyl transferase (cat) gene was initiated by a promoter of either the epothilone operon promoter (epop) or the kanamycin promoter (aphII). These two primers are recognizable in E. coli and $M$. xanthus $[10,11,17]$. The 1.6-kb NdeI-KpnI fragment epop-cat was amplified from the pKK843 plasmid [17] using the CM-EPOP primer pair, while the 1.1-kb NdeI-KpnI fragment aphII-cat was amplified from pKK-aphII [17] using the CM-APH primer pair. The epop-cat and aphII-cat were ligated to the $p 15 \mathrm{~A}$ replicon, generating the vectors of p15A-epop-cat and p15A-aph-cat, respectively. The 2.0-kb transposon element IR-Tpase-IR was amplified from plasmid pMiniHimar-lacZ [13] using the TPASE prime pair, while the $3.0-\mathrm{kb}$ site-specific recombination 
Table 1 Bacterial strains and plasmids used in this study

\begin{tabular}{|c|c|c|}
\hline $\begin{array}{l}\text { Strain and } \\
\text { plasmid }\end{array}$ & Relevant characteristic(s) & $\begin{array}{l}\text { Source or } \\
\text { reference }\end{array}$ \\
\hline \multicolumn{3}{|l|}{ Strain } \\
\hline \multicolumn{3}{|l|}{ E. coli } \\
\hline $\mathrm{DH} 5 \alpha$ & $\mathrm{F}^{-}, \sup \mathrm{E} 44, \Delta \mathrm{lacU} 169$ ( $\varphi 80$ lacZ $\left.\Delta \mathrm{M} 15\right)$, hsdR17, recA1, endA1, gyrA96, thi-1, relA1 & Life technologies \\
\hline GB05-red & $\begin{array}{l}\text { An } E \text {. coli DH10B derive by deletion of fhuA, ybcC and recET, and insertion of } \\
\text { the } P_{\text {BAD }} \text { gbaA cassette at the } y b c C \text { locus }\end{array}$ & {$[25]$} \\
\hline \multicolumn{3}{|l|}{ S. cellulosum } \\
\hline So0157-2 & Epothilone producing strain & This lab \\
\hline \multicolumn{3}{|l|}{ M.xanthus } \\
\hline DK1622 & Wild type & {$[36,37]$} \\
\hline $\mathrm{DZ2}$ & Wild type & {$[38]$} \\
\hline SW504 & dif mutant, contains a difA in frame deletion & {$[22,23]$} \\
\hline \multicolumn{3}{|l|}{ Plasmid } \\
\hline pACYC184 & p15A ori; $\mathrm{Cm}^{r}$ & Novagen \\
\hline pKK843 & $\begin{array}{l}\text { With an 843-bp epothione promoter epop inserted in front of the promoter-less cat } \\
\text { gene (epop-cat); Cm }\end{array}$ & {$[17]$} \\
\hline pKK-aphll & $\begin{array}{l}\text { With an aphl/ promoter fragment inserted in front of the promoter-less cat } \\
\text { gene (aphll-cat); } \mathrm{Cm}^{r}\end{array}$ & {$[17]$} \\
\hline pMiniHimar-lacZ & Himar1 transposon (IR-Tpase-IR), lacZ; Km ${ }^{r}$ & {$[13,14]$} \\
\hline pSWU30 & $\begin{array}{l}\text { Site specific integration vector with } M \times 8 \text { attB } \\
\text { integration site }(M \times 8) ; \text { Tet }^{r}\end{array}$ & {$[27]$} \\
\hline p15A-epop-cat & Ligating the epop-cat to the p15A ori; $\mathrm{Cm}^{r}$ & This study \\
\hline p15A-aph-cat & Ligating the aphll-cat to the p15A ori; $\mathrm{Cm}^{r}$ & This study \\
\hline pTp-epop & Ligating the IR-Tpase-IR to p15A-epop-cat; $\mathrm{Cm}^{r}$ & This study \\
\hline pTp-aph & Ligating the IR-Tpase-IR to p15A-aph-cat; $\mathrm{Cm}^{r}$ & This study \\
\hline pMх8-epop & Ligating Mx8 to p15A-epop-cat; $\mathrm{Cm}^{r}$ & This study \\
\hline pMx8-aph & Ligating Mx8 to p15A-aph-cat; $\mathrm{Cm}^{r}$ & This study \\
\hline Cosmid10 & $\begin{array}{l}\text { A plasmid from a Cosmid library of So0157-2 genome, containing foremost part } \\
\text { of epothilone gene cluster (epoA, epoP, epoB, epoC and part of epoD); Ampr', Neor }\end{array}$ & This study \\
\hline Fosmid3B11 & $\begin{array}{l}\text { A plasmid from a fosmid library of So0157-2 genome, containing latter part of epothilone } \\
\text { gene cluster (partial epoC, epoD, epoE, epoF); Cmr }\end{array}$ & This study \\
\hline pSL1180 & Cloning vector; $A m p^{r}$ & Pharmacia \\
\hline pSL-UCD & $\begin{array}{l}\text { Ligating the 2.0-kb epoup fragment, 2.2-kb epoCov fragment, and the 2.2-kb epodown } \\
\text { fragment into pSL1180; Ampr }\end{array}$ & This study \\
\hline pBJ113 & Gene replacement vector with $\mathrm{KG}$ cassette; $\mathrm{Km}^{\mathrm{r}}$ & [28] \\
\hline pGEM-Teasy & Cloning vector, Ampr & Promega \\
\hline pGEM-galk & Ligating the 1.7-kb galk gene to pGEM-Teasy; Ampr & This study \\
\hline pSL-ETG & Ligating the 3.2-kb TG cassette to pSL-UCD; Ampr', Tet ${ }^{r}$ & This study \\
\hline pCVD442 & A suicide plasmid; Ampr & {$[29]$} \\
\hline pSL-ETGS & Ligating the 1.8-kb sacB gene to pSL-ETG; Amp ${ }^{r}$, Tet $^{r}$ & This study \\
\hline pSET152 & Conjunction Vector; Aprar & This study \\
\hline p15A-apra-cm & Ligating 1.7-kb apra fragment to p15A-aph-cat; Aprar', Cmr & This study \\
\hline p15A-apra-cm-Tp & Ligating IR-Tpase-IR to p15A-apra-cm; Apra', Cmr & This study \\
\hline p15A-apra-cm-Mx8 & Ligating M×8 to p15A-apra-cm; Aprar', Cmr & This study \\
\hline p15A-recTp & Ligating the 11.5-kb Munl-Spel fragment from pSL-ETGS to p15A-apra-cm-Tp; Aprar', Cmr, Tet' & This study \\
\hline p15A-recMx8 & Ligating the 11.5-kb Munl-Spel fragment from pSL-ETGS to p15A-apra-cm-Mx8; Aprar', Cmr, Tet ${ }^{r}$ & This study \\
\hline p15A-TP-fos & Recombination between the linearized Fosmid3B11 and p15A-recTp; Apra', Cmr, Tet ${ }^{r}$ & This study \\
\hline p15A-Mx8-fos & Recombination between the linearized Fosmid3B11 and p15A-recMx8; Apra', Cmr, Tet ${ }^{r}$ & This study \\
\hline p15A-Tp-CF & Recombination between the linearized Cosmid10 and p15A-TP-fos; Aprar', Cmr & This study \\
\hline p15A-Mx8-CF & Recombination between the linearized Cosmid10 and p15A-TP-fos; Aprar', Cmr & This study \\
\hline
\end{tabular}


element $M x 8$ was generated from the template pSWU30 [27] using the MX8 primer pair. Finally, the plasmids pTp-epop (or pTp-aph) and pMx8-epop (or pMx8aph) were constructed by ligating the IR-Tpase-IR and $M x 8$ to p15A-epop-cat (or p15A-aph-cat), respectively, which had been digested with NdeI; and the DNA ends were blunted using the T4 DNA polymerase. The PCR primer pairs used in this study are summarized in Additional file 5: Table S3.

\section{Stitching and recombination of epothilone gene cluster}

We constructed cosmid and fosmid libraries of Sorangium cellulosum So0157-2 genome. Southern blotting hybridization with epothilone gene probes revealed clones containing the epothilone genes. The harbored epothilone genes fragments in the positive clones were determined by the end sequencing. We selected Cosmid10 and Fosmid3B11 for our construction. After completely sequencing, Cosmid10 contained a fragment of complete epoA to epoC and part of epoD, while Fosmid3B11 contained part of epoC till the downstream sequence of epoF.

To stitch the two epothilone-genes fragments in Cosmid10 and Fosmid3B11, transitional recombinant vectors $\mathrm{p} 15 \mathrm{~A}-$ recT and $\mathrm{p} 15 \mathrm{~A}-\mathrm{recM}$ were constructed as follows. First, the 2.0-kb BglII-XbaI epoup fragment (upstream of epoA), 2.2-kbXbaI-NdeI epoCov fragment (overlapping the epoC sequence), and the 2.2-kb NotI-SpeI epodown fragment (downstream of epoF) were amplified from the genomic DNA of S. cellulosum So0157-2 using the primer pairs of UP, C-LAP and DOWN, respectively. The three PCR products, used as the homologous arms for recombination, were ligated serially into the corresponding restriction sites of pSL1180, generating pSL-UCD. Then, the $1.7-\mathrm{kb}$ galk gene, containing $\mathrm{XbaI}$ and NheI sites at the $5^{\prime}$ end and XbaI site at the $3^{\prime}$ end, was amplified from $\mathrm{pBJ} 113$ [28] using the GALK primer pair, which was further ligated into the XbaI site of pGEM-Teasy vector, generating pGEM-galk. The $1.5-\mathrm{kb}$ NheI tet fragment (tetracycline gene), amplified from pSWU30 using the TET primer pair, was ligated into the NheI site of the galk fragment in pGEM-galk. The 3.2-kb tet-galk fragment (TG cassette), used for positive and negative selection, was cleaved with Xba I, and ligated into the $\mathrm{XbaI}$ site of pSL-UCD, generating pSL-ETG. The plasmid pSL-ETG was cleaved with NdeI and ligated with the 1.8-kb Nde I $s a c B$ gene, which was amplified from pCVD442 [29] using the SACB primer pair, producing pSL-ETGS.

In another route, a 1.7-kb apra fragment (apramycin gene), amplified from pSET152 [30] using the APRA primer pair, was cloned into NdeI site of p15A-aph-cat to create p15A-apra-cm. Then, the PCR fragment IR-Tpase$I R$ or $M x 8$ was ligated into $\mathrm{p} 15 \mathrm{~A}-\mathrm{apra}-\mathrm{cm}$, which thereafter was cleaved at the $3^{\prime}$ end of the cat gene by ScaI, yielding the plasmid p15A-apra-cm-Tp and p15A-apra$\mathrm{cm}-\mathrm{Mx} 8$, respectively. Finally, the 11.5-kb MunI-SpeI fragment from pSL-ETGS with the blunt ends was cloned into the p15A-apra-cm-Tp, which had been cleaved with SnaBI with a blunt end, to create the purposed vector p15A- recTp. The plasmid was cloned into p15A-apracm-Mx8, which was further cleaved with KpnI, forming the blunt ends, to create the other purposed vector p15A-recMx8.

The vectors $\mathrm{p} 15 \mathrm{~A}-\mathrm{recTp}$ and $\mathrm{p} 15 \mathrm{~A}-\mathrm{recMx} 8$ was subsequently engineered for recombining with the epothilone gene cluster from Cosmid10 and Fosmid3B11. First, both Cosmid10 and Fosmid3B11 were cleaved with DraI, removing the irrelevant sequences and exposing the terminal sequences with epothilone genes, which contained the identical homologous arms epoup, epoCov and epodown. Then, the linearized Fosmid3B11 was electroporated into the $E$. coli strain GB05-red harboring p15A-recTp or p15A-recMx8 for the first round of recombination and the transformants with the objective recombinant p15A-TP-fos or p15AMx8-fos were selected on the LB plate containing Apra and $\mathrm{Cm}$ with extra $10 \%$ sucrose for negative selection. Similarly, in the second round the linearized Cosmid10 was electroporated into E. coli GB05-red harboring the plasmid from the first round and the transformants were selected on the LB plate containing extra 1\% 2-deoxygalactose (DOG). Ultimately, the engineered recombinant plasmids $\mathrm{p} 15 \mathrm{~A}-\mathrm{Tp}-\mathrm{CF}$ and $\mathrm{p} 15 \mathrm{~A}-\mathrm{Mx} 8-\mathrm{CF}$ containing the integrated epothilone gene cluster were constructed. All the above PCR products were amplified using the $p f u$ DNA polymerase to ensure high fidelity and sequenced to confirm their identities. A diagrammatic sketch for the construction is provided in Figure 2.

\section{Electro-transformation of $M$. xanthus}

The constructed expression plasmids were introduced into $M$. xanthus cells by electroporation. Briefly, $M$. xanthus cells from $50-\mathrm{mL}$ overnight cultures $\left(\mathrm{OD}_{600}\right.$ was about 0.6) were collected and washed thrice with ice-cold water for preparing competent cells. $1.5 \mathrm{~mL}$ of the culture was centrifuged and the precipitate was resuspended with $50 \mu \mathrm{L}$ of ice-cold water in tube, mixed with $3 \mu \mathrm{g}$ of DNA, and electroporated at a voltage of $1,250 \mathrm{~V}$ in a $2 \mathrm{~mm}$ cuvette using the Electroporator (Eppendorf, Germany). Then the cells were transferred into $2 \mathrm{~mL}$ CYE liquid medium in a $10-\mathrm{mL}$ tube and incubated at $30^{\circ} \mathrm{C}$ with a rotate speed of $250 \mathrm{rpm}$. After $4-6 \mathrm{~h}$ of incubation, $0.1-0.5 \mathrm{~mL}$ of cells 
was added to $2 \mathrm{~mL} 0.5 \%$ soft agar and the mixture was spread on 1.5\% CYE selection agar plates. Resistant colonies that appeared after 6 days of incubation were checked by colony PCR with the following primers: primer pairs of CM-APH and CM-EPOP were used to check the integration of cat gene; Epothilone-specific primers, EA, EP, EB, EC1, EC2, EE and EF, with a 51\% coverage of the whole gene cluster, were designed to detect the regions located in ORFs epoA, epoP, epoB, epoC, epoE or epoF, respectively, to verify the integration of the whole biosynthetic gene cluster in the $M$. xanthus chromosome.

\section{Measurement of chloramphenicol acetyl transferase (CAT) activity in $M$. xanthus}

To measure the chloramphenicol acetyl transferase (CAT) activity, the bacterial pellets were harvested by centrifugation at $8,000 \times g$ for $5 \mathrm{~min}$ after the cultures reached the mid-exponential phase (36 h of incubation; $\mathrm{OD}_{600}$ was about 3.0). The pellet was resuspended and washed twice with distilled water and then resuspended in $1 \mathrm{~mL}$ of lysis buffer. The crude bacterial extracts were prepared by sonication, and the supernatant was collected by centrifugation at $12,000 \times g$ for $2 \mathrm{~min}$, and used for characterizing chloramphenicol acetyl transferase (cat) gene expression activity with the CAT ELISA kit, as previously described [17].

\section{Epothilone extraction and detection}

M. xanthus strains containing the complete epothilone gene cluster were grown overnight in $100 \mathrm{~mL}$ CYE medium supplemented with Apra and $\mathrm{Cm}$ antibiotics. The cultures were inoculated at a ratio of 2:100 into CYE medium containing $2 \%$ of the XAD-16 resin for the absorption of epothilone products. After 7 days rotation at the $250 \mathrm{rpm}$ speed and $30^{\circ} \mathrm{C}$, the mixtures of cells and resin were harvested by centrifugation and extracted with two volume of methanol by shaking at room temperature overnight [31]. After centrifuged for $20 \mathrm{~min}$, the supernatant was moved into a rotary evaporator to remove the solvent. The residue was further re-dissolved in $1 \mathrm{~mL}$ of methanol, and an aliquot of $20 \mu \mathrm{L}$ of the sample was injected into a Finnigan HPLC system interfaced with a Finnigan MSQ classic quadrupole mass spectrometer (Thermo Finnigan, USA). The analysis was carried out on a Shim-pack MRC-ODS RP column $(4.6 \mathrm{~mm} \times 250 \mathrm{~mm}, 4.60 \mu \mathrm{m}$; Shimadzu, Japan) at a temperature of $28^{\circ} \mathrm{C}$ with a mobile phase of $60 \%$ methanol (HPLC grade) and $40 \%$ buffer $(0.2 \%$ acetate acid/18 M $\Omega$ Millipore water) at a flow rate of $1.0 \mathrm{~mL} /$ min. The MS analysis was performed under the following conditions: ESI-positive, probe temperature of $450^{\circ} \mathrm{C}$, cone voltage of $75 \mathrm{~V}$, full scan mass range from
200 to $2,000 \mathrm{~m} / \mathrm{z}$ at $2 \mathrm{~Hz}$ scan speed, and SIM scan at 494 $[\mathrm{M}+\mathrm{H}]^{+}$for epothilone $\mathrm{A}$ and $508[\mathrm{M}+\mathrm{H}]^{+}$for epothilone B [32]. Epothilones were identified by comparison to the retention time, $249 \mathrm{~nm}$ of UV spectra and the $\mathrm{MS}^{2}$ pattern of the authentic reference standard sample containing epothilones $\mathrm{A}$ and $\mathrm{B}$, which were obtained from the culture of S. cellulosum So0157-2 as reported previously $[31,33]$. The production levels of epothilones were averaged from three independent cultivations and extractions.

\section{Determination of the integration sites}

A Genome Walking kit (TaKaRa, Japan) was used to verify the insertion sites of the gene integration. Three specific primers, $\mathrm{Cm} 1, \mathrm{Cm} 2$ and $\mathrm{Cm} 3$, designed in this work (Additional file 5: Table S3) were employed with the random primer AP1, which is provided by the kit, in three PCR rounds, to amplify a product containing partial cat region, the IR sequence of the transposon and the flanking unknown genome region of $M$. xanthus, respectively. Genomic DNA of the transformants with the epothilone gene cluster was extracted and used as the template in the first round PCR using the primer pair AP1/Cm1; the diluted product from the first PCR round was orderly used as the template for the second round, using the primer pair AP1/Cm2; and the product from the second round was diluted as the template for the third PCR round, using the AP1/Cm3 primer pair. The PCR systems and procedures were performed according to the kit protocol. The PCR products were detected by electrophoresis, and the single DNA bands with appropriate size from the third PCR round were withdrawn by the DNA Extraction kit (Promega, USA) and sequenced after subcloning to the pGEM-Teasy vector.

\section{Transcriptomics analysis}

In this study, we exploit the ssRNA-seq method to identify the transcriptional template strands of $M$. xanthus DZ2 and three transformants ZE-5, ZE-9 and ZE-14 at a whole genome level using the Illumina-platform high throughput sequencing. First of all, the total RNA of the $M$. xanthus strains were extracted according to the protocol provided by the SV Total RNA isolation system kit (Promega, USA). Residual genomic DNA was removed by treatment with recombinant DNase I (RNase-free; Ambion, USA) according to the manufacturer's instructions. The quality of the total RNA was verified by agarose gel electrophoresis, and the concentration was determined using a NanoDrop ND-1000 spectrophotometer (NanoDrop technologies, USA). Then, libraries were created by modifying the previously described dUTP second strand method [34]. We fragmented 
200 ng of $M$. xanthus polyA ${ }^{+}$RNA by heating at $98^{\circ} \mathrm{C}$ for $40 \mathrm{~min}$ in $0.2 \mathrm{mM}$ sodium citrate, $\mathrm{pH}$ 6.4 (Ambion, USA). The fragmented RNA was concentrated to $5 \mu \mathrm{L}$, mixed with $3 \mu \mathrm{g}$ random hexamers, incubated at $70^{\circ} \mathrm{C}$ for $10 \mathrm{~min}$, and then cooled on ice. The RNA mixtures were further added with $4 \mu \mathrm{L}$ of $5 \times$ first-strand buffer, $2 \mu \mathrm{L}$ of $100 \mathrm{mM}$ DTT, $1 \mu \mathrm{L}$ of $10 \mathrm{mM}$ dNTPs, $4 \mu \mathrm{g}$ of actinomycin D (USB), 200 U SuperScript III, and $20 \mathrm{U}$ SUPERase-In (Ambion, USA), incubated at room temperature for $10 \mathrm{~min}$ followed by $1 \mathrm{~h}$ at $55^{\circ} \mathrm{C}$ to synthesize the first-strand cDNA. First-strand cDNA was cleaned up by extraction twice with phenol: chloroform: isoamyl alcohol (25:24:1), followed by ethanol precipitation with 0.1 volumes $5 \mathrm{M}$ ammonia acetate to remove dNTPs and re-suspension in $104 \mu \mathrm{L} d \mathrm{ddH}_{2} \mathrm{O}$. Secondstrand cDNA was synthesized by adding $4 \mu \mathrm{L} 5 \times$ firststrand buffer, $2 \mu \mathrm{L} 100 \mathrm{mM}$ DTT, $4 \mu \mathrm{L} 10 \mathrm{mM}$ dNTPs with dTTP replaced by dUTP (Sigma-Aldrich, USA), $30 \mu \mathrm{L} 5 \times$ second strand buffer, $40 \mathrm{U}$ Escherichia coli DNA polymerase, $10 \mathrm{U}$ E. coli DNA ligase, $2 \mathrm{U}$ E. coli RNase $\mathrm{H}$ and incubating at $16^{\circ} \mathrm{C}$ for $2 \mathrm{~h}$. A paired-end library for Illumina sequencing was prepared according to the instructions provided with the following modifications. First, five times less adapter mixture was ligated to the cDNAs. Second, 1 U USER (New England Biolabs, USA) was incubated with 180- to 480-bp size-selected, adapter-ligated $\mathrm{cDNA}$ at $37^{\circ} \mathrm{C}$ for $15 \mathrm{~min}$ followed by $5 \mathrm{~min}$ at $95^{\circ} \mathrm{C}$ before PCR. Third, PCR was performed with Phusion High-Fidelity DNA Polymerase with GC buffer (New England Biolabs, USA) and $2 \mathrm{M}$ betaine (Sigma, USA). Fourth, PCR primers were removed using $1.8 \times$ volume of AMPure PCR Purification kit (BeckmanCoulter Genomics, USA). Transcriptome sequencing was performed at the BGI Corporation. Reagents were all from Invitrogen (Carlsbad, USA) except as noted.

To assay the growth curves, $M$. xanthus colonies were first cultured overnight in CYE medium. Then, the cultures were transferred at a ratio of about 2:100 into $5 \mathrm{~mL}$ fresh CYE medium with a start $\mathrm{OD}_{600}$ of 0.15 , and assayed of the $\mathrm{OD}_{600}$ values periodically.

The reference genome (M. xanthus DK1622) and the gene annotation was retrieved from GenBank (accession no. NC_008095.1). After removing the reads containing sequencing adapters and low-quality reads (reads containing Ns $>10 \%$ ), the remaining 90 bp clean reads with high quality were aligned with the reference genome using Bowtie software [35]. Then the RPKM method was used to normalize the transcript level, which was expressed as the number of reads per kilobase of exon region per million mapped reads (RPKM). Go annotation of the genes was performed using Blast2GO software and visualized by WEGO software.

\section{Additional files}

Additional file 1: Figure S1. Diagram for the construction of the CAT reporter gene vectors. The epop and aphll promoters were linked to p15A to generate p15-epop-cat and p15A-aph-cat, respectively. Then, the IRTpase-IR or Mx8 attp element was subcloned to each of the two plasmids above, respectively, to build the four final plasmids pTp-epoP, pM×8epoP, pTp-aph and pMx8-aph, which were initiated by aphll and epoP, respectively. The plasmids were electroporated into $M$. xanthus to assay the promoter activities.

Additional file 2: Table S1. The insertion sites of the epothilone genes and the production of epothilones in different transposition recombinants.

Additional file 3: Figure S2. PCR amplification of the junction regions between the seven epothilone-modules. Genome templates for each PCR sample are as follows: 1, M. xanthus DZ2; 2, M. xanthus ZE5; 3 , M. xanthus ZE9; 4, M. xanthus ZE14; 5, S. cellulosum So0157-2; 6, distilled water; M, DNA Marker DL2000 plus II.

Additional file 4: Table S2. Significantly up- and down-regulated Myxococcus genes in different recombinants.

Additional file 5: Table S3. Primers used in this study.

\section{Authors' contributions}

$L P Z, Y Z L$ designed the experiments. LPZ, XJY, KH analyzed and interpreted the data. $L P Z, X J Y, Z F L, L S Z, X N Y, H L W$ performed the experiments. $L P Z, Y Z L$ wrote the manuscript. YMZ, YZL supervised the research. All authors read and approved the final manuscript.

\section{Acknowledgements}

This work was financially supported by the National Science Foundation of China (NSFC) Key Program [No. 31130004] and National High-tech R\&D Program of China (863 Program) [No. 2012AA02A701]. We thank Chunyan Lin and Guang-rong Zhao from Tianjin University for exchanged communications.

\section{Compliance with ethical guidelines}

\section{Competing interests}

The authors declare that they have no competing interests.

Received: 17 May 2015 Accepted: 7 July 2015

Published online: 22 July 2015

\section{References}

1. Rosano GL, Ceccarelli EA (2014) Recombinant protein expression in Escherichia coll: advances and challenges. Front Microbiol. 5, article 172

2. Ross AC, Gulland LE, Dorrestein PC, Moore BS (2015) Targeted capture and heterologous expression of the Pseudoalteromonas alterochromide gene cluster in Escherichia coli represents a promising natural product exploratory platform. ACS Synth Biol. doi:10.1021/sb500280q

3. Wang Y, Li YZ (2014) Cultivation to improve in vivo solubility of overexpressed arginine deiminases in Escherichia coli and the enzyme characteristics. BMC Biotechnol 14:53. doi:10.1186/1472-6750-14-53

4. Ongley SE, Bian X, Neilan BA, Müller R (2013) Recent advances in the heterologous expression of microbial natural product biosynthetic pathways. Nat Prod Rep 30:1121-1138

5. Gerth K, Bedorf N, Höfle G, Irschik H, Reichenbach H (1996) Epothilons A and B: antifungal and cytotoxic compounds from Sorangium cellulosum (Myxobacteria). Production, physico-chemical and biological properties. J Antibiot (Tokyo) 49:560-563

6. Tang L, Shah S, Chung L, Carney J, Katz L, Khosla C et al (2000) Cloning and heterologous expression of the epothilone gene cluster. Science 287:640-642 
7. Zhang L, Zhao GP, Ding XM (2011) Tandem assembly of the epothilone biosynthetic gene cluster by in vitro site-specific recombination. Sci Rep Uk. doi:10.1038/srep00141

8. Park SR, Park JW, Jung WS, Han AR, Ban YH, Kim EJ et al (2008) Heterologous production of epothilones B and D in Streptomyces venezuelae. Appl Microbiol Biotechnol 81:109-117

9. Mutka SC, Carney JR, Liu Y, Kennedy J (2006) Heterologous production of epothilone C and D in Escherichia coli. Biochemistry 45:1321-1330

10. Fu J, Wenzel S, Perlova O, Wang J, Gross F, Tang Z et al (2008) Efficient transfer of two large secondary metabolite pathway gene clusters into heterologous hosts by transposition. Nucleic Acids Res 36(17):e113

11. Julien B, Shah S (2002) Heterologous expression of epothilone biosynthetic genes in Myxococcus xanthus. Antimicrob Agents Chemother 46:2772-2778

12. Tang L, Chung L, Carney JR, Starks CM, Licari P, Katz L (2005) Generation of new epothilones by genetic engineering of a polyketide synthase in Myxococcus xanthus. J Antibiot (Tokyo) 58:178-184

13. Bouhenni R, Gehrke A, Saffarini D (2005) Identification of genes involved in cytochrome c biogenesis in Shewanella oneidensis, using a modified mariner transposon. Appl Environ Microbiol 71:4935-4937

14. Zhang CY, Cai K, Liu H, Zhang Y, Pan HW, Wang B et al (2007) New locus important for Myxococcus social motility and development. J Bacteriol 189:7937-7941

15. Tojo N, Sanmiya K, Sugawara H, Inouye S, Komano T (1996) Integration of bacteriophage Mx8 into the Myxococcus xanthus chromosome causes a structural alteration at the C-terminal region of the IntP protein. J Bacteriol 178:4004-4011

16. Viswanathan P, Murphy K, Julien B, Garza AG, Kroos L (2007) Regulation of dev, an operon that includes genes essential for Myxococcus xanthus development and CRISPR-associated genes and repeats. J Bacteriol 189:3738-3750

17. Zhu LP, Li ZF, Sun X, Li SG, Li YZ (2013) Characteristics and activity analysis of epothilone operon promoters from Sorangium cellulosum strains in Escherichia coli. Appl Microbiol Biotechnol 97:6857-6866

18. Hao T, Biran D, Velicer GJ, Kroos L (2002) Identification of the Omega4514 regulatory region, a developmental promoter of Myxococcus xanthus that is transcribed in vitro by the major vegetative RNA polymerase. J Bacteriol 184:3348-3359

19. Zhang C, Cai K, Pan H, Liu H, Li Y (2010) Construction and application of plasmid pZCY11 for analyzing gene functions and expressions in Myxococcus. Wei Sheng Wu Xue Bao. 50:29-35

20. Julien B, Shah S, Ziermann R, Goldman R, Katz L, Khosla C (2000) Isolation and characterization of the epothilone biosynthetic gene cluster from Sorangium cellulosum. Gene 249:153-160

21. Molnar I, Schupp T, Ono M, Zirkle RE, Milnamow M, Nowak-Thompson B et al (2000) The biosynthetic gene cluster for the microtubule-stabilizing agents epothilones A and B from Sorangium cellulosum So ce90. Chem Biol 7:97-109

22. Yang Z, Geng Y, Xu D, Kaplan HB, Shi W (1998) A new set of chemotaxis homologues is essential for Myxococcus xanthus social motility. Mol Microbiol 30:1123-1130
23. Wang J, Hu W, Lux R, He X, Li Y, Shi W (2011) Natural transformation of Myxococcus xanthus. J Bacteriol 193:2122-2132

24. Li PF, Li SG, Li ZF, Zhao L, Wang T, Pan HW et al (2013) Co-cultivation of Sorangium cellulosum strains affects cellular growth and biosynthesis of secondary metabolite epothilones. FEMS Microbiol Ecol 85:358-368

25. Fu J, Bian X, Hu S, Wang H, Huang F, Seibert PM et al (2012) Full-length RecE enhances linear-linear homologous recombination and facilitates direct cloning for bioprospecting. Nat Biotechnol 30:440-446

26. Goldman B, Bhat S, Shimkets LJ (2007) Genome evolution and the emergence of fruiting body development in Myxococcus xanthus. PLoS One 2:e1329

27. Wu SS, Wu J, Kaiser D (1997) The Myxococcus xanthus pilT locus is required for social gliding motility although pili are still produced. Mol Microbiol 23:109-121

28. Julien B, Kaiser AD, Garza A (2000) Spatial control of cell differentiation in Myxococcus xanthus. Proc Natl Acad Sci U S A. 97:9098-9103

29. Xia ZJ, Wang J, Hu W, Liu H, Gao XZ, Wu ZH et al (2008) Improving conjugation efficacy of Sorangium cellulosum by the addition of dual selection antibiotics. J Ind Microbiol Biotechnol 35:1157-1163

30. Wilkinson CJ, Hughes-Thomas ZA, Martin CJ, Bohm I, Mironenko T, Deacon M et al (2002) Increasing the efficiency of heterologous promoters in actinomycetes. J Mol Microbiol Biotechnol 4:417-426

31. Gong GL, Sun X, Liu XL, Hu W, Cao WR, Liu H et al (2007) Mutation and a high-throughput screening method for improving the production of Epothilones of Sorangium. J Ind Microbiol Biotechnol 34:615-623

32. Li SG, Zhao L, Han K, Li PF, Li ZF, Hu W et al (2014) Diversity of epothilone producers among Sorangium strains in producer-positive soil habitats. Microb Biotechnol 7:130-141

33. Perlova O, Fu J, Kuhlmann S, Krug D, Stewart AF, Zhang Y et al (2006) Reconstitution of the myxothiazol biosynthetic gene cluster by Red/ET recombination and heterologous expression in Myxococcus xanthus. Appl Environ Microbiol 72:7485-7494

34. Parkhomchuk D, Borodina T, Amstislavskiy V, Banaru M, Hallen L, Krobitsch S et al (2009) Transcriptome analysis by strand-specific sequencing of complementary DNA. Nucleic Acids Res 37:e123

35. Langmead B, Salzberg SL (2012) Fast gapped-read alignment with Bowtie 2. Nat Methods 9:357-359

36. Kaiser D (1979) Social gliding is correlated with the presence of pili in Myxococcus xanthus. Proc Natl Acad Sci USA 76:5952-5956

37. Wang Y, Zhang WY, Zhang Z, Li J, Li ZF, Tan ZG et al (2013) Mechanisms involved in the functional divergence of duplicated GroEL chaperonins in Myxococcus xanthus DK1622. PLoS Genet 9:e1003306

38. Müller S, Willett JW, Bahr SM, Darnell CL, Hummels KR, Dong CK et al. (2013) Draft genome sequence of Myxococcus xanthus wild-type strain DZ2, a model organism for predation and development. Genome Announc 1: e00217-13

\section{Submit your next manuscript to BioMed Central and take full advantage of:}

- Convenient online submission

- Thorough peer review

- No space constraints or color figure charges

- Immediate publication on acceptance

- Inclusion in PubMed, CAS, Scopus and Google Scholar

- Research which is freely available for redistribution

Submit your manuscript at 